\title{
氰基在药物分子设计中的应用
}

\author{
王 江 柳 红* \\ (中国科学院上海药物研究所新药研究国家重点实验室 上海 201203)
}

\begin{abstract}
摘要 含氧基药物在临床治疗药物中占有相当比重. 将氧基基团引入到小分子药物中是药物化学结构改造的重要研究 策略之一. 综述了氰基在药物分子设计中的应用, 氧基的引入可以调节小分子药物的物理化学特性, 改变小分子的药 代动力学性质, 提高药物的生物利用度; 通过氢键相互作用、共价相互作用、偶极相互作用以及 $\pi-\pi$ 相互作用增强配体 与靶标蛋白的相互结合能力以及对其它靶标蛋白的选择性; 同时, 㲵基是㛶基、卤素等多种官能团的生物电子等排体; 将氨基引入到药物小分子当中, 可以通过阻断易代谢位点进而提高药物代谢稳定性等.
\end{abstract}

关键词 㲵基; 药物设计; 结构优化

\section{Application of Nitrile in Drug Design}

\author{
Wang, Jiang Liu, Hong* \\ (State Key Laboratory of Drug Research, Shanghai Institute of Materia Medica, \\ Chinese Academy of Sciences, Shanghai 201203)
}

\begin{abstract}
Nitrile-containing compounds comprise a substantial proportion in the therapeutic drugs. It is an important strategy to introduce nitrile substitute in the small molecule for structure-based medicinal chemistry. The application of nitrile in drug design is reviewed. The nitrile can modulate the physicochemical and pharmacokinetic properties to improve bioavailability, enhance the selectivities and binding affinity to target proteins by hydrogen bond interactions, covalent interactions, polar interactions, and $\pi-\pi$ interactions. Meanwhile, the nitrile acts as bioisostere of various functional groups such as carbonyl and halogen groups. In addition, introducing the nitrile to drug molecules can block metabolically labile sites to increase the metabolic stability of drugs.
\end{abstract}

Keywords nitrile; drug design; lead optimization

目前, 许多临床使用的药物中都含有氧基基团. 氧 基是具有较强极性的碳氮参键, 具有较强的吸电子性 质, 其体积仅为甲基的 $1 / 8$, 因此, 氧基能够深入到靶标 蛋白深处与活性部位的关键氨基酸残基形成氢键相互 作用. 氧基还是良好的氢键受体, 通常与靶标蛋白中的 丝氨酸和精氨酸形成氢键相互作用; 同时, 氧基是羰 基、卤素等多种官能团的生物电子等排体, 将氞基引入 到药物小分子当中, 能够改变小分子的物理化学性质, 增强药物小分子与靶标蛋白的相互作用, 提高药效; 氧 基还可以作为代谢阻断位点, 抑制小分子发生氧化代 谢，提高化合物在体内的代谢稳定性. 鉴于以上特点， 氧基基团已经被广泛应用于药物小分子的结构修饰与 改造中, 药物设计中的氰基取代策略已经成为先导化合 物结构优化的重要研究策略之一 ${ }^{[1]}$. 目前, 临床应用的
含氧基代表药物包括抗糖尿病药物二肽基肽酶 IV 抑制

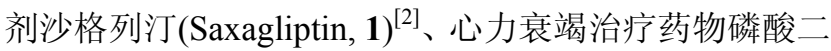
酯酶抑制剂米力农(Milrinone, 2) ) $^{[3]}$ 、降血压药物钻通道 拮抗剂维拉帕米(Verapamil, 3) ${ }^{[4]}$ 、痛风性关节炎治疗药 物黄嘌呤氧化酶抑制剂非布索坦(Febuxostat, 4) ${ }^{[5]}$ 、艾滋 病治疗药物非核苷逆转录酶抑制剂依曲韦林(Etravirine, 5) ${ }^{[6]}$ 以及前列腺癌治疗药物雄激素受体拮抗剂比卡鲁胺 (Bicalutamide, 6) ${ }^{[7]}$ 等(Chart 1).

基于氧基在药物设计中的重要应用, 本文主要综述 了氧基在改变小分子理化性质; 通过氢键作用、共价键 作用、偶极相互作用和 $\pi-\pi$ 相互作用增强与靶标蛋白的 结合能力; 作为羰基、甾素等多种官能团的生物电子等 排体进行小分子结构优化; 以及提高代谢稳定性等几个 方面在药物设计中的应用.

*E-mail: hliu@mail.shcnc.ac.cn

Received February 13, 2012; revised March 30, 2012; published online April 27, 2012.

Project supported by the National Natural Science Foundation of China (No. 81025017).

国家杰出青年科学基金(No. 81025017)资助项目. 
<smiles>N#C[C@@H]1[C@H]2C=C[C@@H](C2)N1C(=O)[C@@H](N)C12CC3CC(CC(O)(C3)C1)C2</smiles>

沙格列汀 (1)<smiles>Cc1nc(-c2ccc(OCC(C)C)c(C#N)c2)sc1C(=O)O</smiles>

非布索坦 (4)<smiles>Cc1[nH]c(=O)c(C#N)cc1-c1ccncc1</smiles>

米力农 (2)<smiles>Cc1cc(C#N)cc(C)c1Oc1nc(Nc2ccc(C#N)cc2)nc(N)c1Br</smiles>

依曲韦林 (5)<smiles>COc1ccc(CCN(C)CCCC(C#N)(c2ccc(OC)c(OC)c2)C(C)C)cc1OC</smiles>

维拉帕米 (3)<smiles>N#Cc1ccc(NC(=O)C(O)(O)CS(=O)(=O)c2ccc(F)cc2)cc1C(F)(F)F</smiles>

比卡鲁胺 $(6)$

Chart 1

\section{1 药物的理化性质}

\section{1 药物的溶解度}

溶解度是化合物在一定溶液条件下的最大溶解浓 度. 药物的溶解度对于药物吸收和口服生物利用度具有 决定性意义. 药物的溶解度与许多因素有关, 其中最重 要的就是化合物的结构, 在药物小分子中适当地引入氰 基基团能够有效地增加药物的溶解度.

法尼基转移酶通过阻断细胞信号传导进而预防肿 瘤发生是重要的抗肿瘤靶点 ${ }^{[8]}$. 施贵宝公司研发的法尼 基转移酶抑制剂(8, BMS-214662)主要用于慢性髓细胞 样白血病的治疗 $\left(\mathrm{IC}_{50}=1.35 \mathrm{nmol} / \mathrm{L}\right)$, 目前处于临床 II 期研究阶段(Eq. 1). 化合物 $\mathbf{8}$ 与法尼基转移酶晶体复合 物模式图表明，该化合物与蛋白之间具有较强的 $\pi-\pi$ 相 互作用(图 1) ${ }^{[9]}$. 虽然氰基没有与特定的氨基酸残基形成 氢键相互作用, 但是氭基改善了该化合物的药代动力学 特性. 溶解性实验表明, 氘基取代与相应的溴代化合物 7 相比, 活性提高了 44 倍, 水溶性提高了近 10 倍 ${ }^{[10]}$.

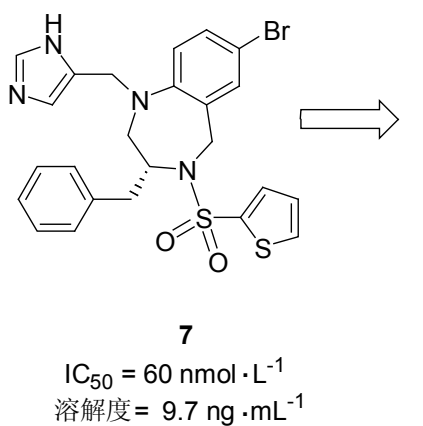<smiles>N#Cc1ccc2c(c1)CN(S(=O)(=O)c1cccs1)[C@@H](Cc1ccccc1)CN2Cc1cnc[nH]1</smiles>

8 (BMS-214662)

$\mathrm{IC}_{50}=1.35 \mathrm{nmol} \cdot \mathrm{L}^{-1}$ 溶解度 $=81 \mathrm{ng} \cdot \mathrm{mL}^{-1}$

\section{2 降低碱性}

由于氰基是较强的吸电子基团，因此，将氰基引入 药物小分子当中能够有效地降低化合物的碱性. Seydel

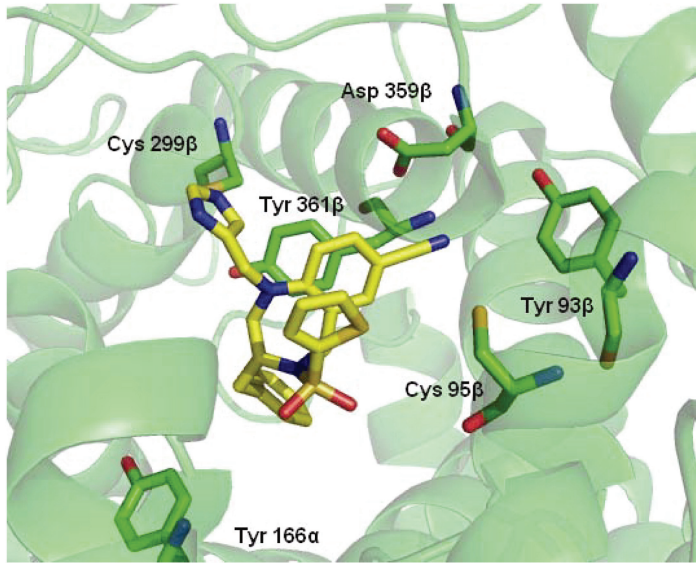

图 1 化合物 8 与法尼基转移酶的单晶结构示意图

Figrue 1 Crystal structure of binding mode of compound 8 to farnesyl transferase

等 ${ }^{[11]}$ 在进行磺胺类药物研发过程中发现，在右侧的苯 环上引入不同的取代基，对化合物的酸碱性以及抗菌活 性影响显著，活性随 $\mathrm{p} K_{\mathrm{a}}$ 的降低而升高(表 1). 其中，在 苯环的 4-位引入氰基取代基，能够显著降低化合物的 $\mathrm{p} K_{\mathrm{a}}$, 化合物 17 的 $\mathrm{p} K_{\mathrm{a}}$ 为 7.36 , 其抗菌活性提高 $(\mathrm{MIC}=$ $1.0 \mu \mathrm{mol} / \mathrm{L}$ ), 与 4-甲氧基取代化合物 9 相比, $\mathrm{p} K_{\mathrm{a}}$ 约降低 2 , 活性提高了近 10 倍.

\section{2 药物的药效}

\section{1 增强蛋白-配体相互作用}

在小分子药物中引入氰基基团，能够有效地增强靶 标蛋白与配体之间的相互作用. 在多种药物小分子的开 发中, 氰基基团的引入都能显著提高小分子与靶标蛋白 之间的结合能力，主要通过氢键相互作用、共价键相互 作用、偶极相互作用以及 $\pi-\pi$ 相互作用等. 
表 1 磺胺类药物的酸碱性和抗菌活性

Table 1 Basicity and antibacterial activities in sulfonamide drugs<smiles>[R]c1ccc(NS(=O)(=O)c2ccc(N)cc2)c([R])c1[R]</smiles>

$9 \sim 18$

\begin{tabular}{cccccc}
\hline 化合物 & $\mathrm{R}^{1}$ & $\mathrm{R}^{2}$ & $\mathrm{R}^{3}$ & $\mathrm{p} K_{\mathrm{a}}$ & $\mathrm{MIC} /\left(\mu \mathrm{mol} \cdot \mathrm{L}^{-1}\right)$ \\
\hline $\mathbf{9}$ & $\mathrm{H}$ & $\mathrm{H}$ & $\mathrm{OMe}$ & 9.34 & 34.50 \\
$\mathbf{1 0}$ & $\mathrm{H}$ & $\mathrm{H}$ & $\mathrm{H}$ & 9.10 & 16.00 \\
$\mathbf{1 1}$ & $\mathrm{H}$ & $\mathrm{H}$ & $\mathrm{Cl}$ & 8.56 & 13.00 \\
$\mathbf{1 2}$ & $\mathrm{H}$ & $\mathrm{H}$ & $\mathrm{I}$ & 8.17 & 11.25 \\
$\mathbf{1 3}$ & $\mathrm{Cl}$ & $\mathrm{H}$ & $\mathrm{OMe}$ & 8.81 & 16.60 \\
$\mathbf{1 4}$ & $\mathrm{H}$ & $\mathrm{CF}_{3}$ & $\mathrm{H}$ & 7.98 & 5.60 \\
$\mathbf{1 5}$ & $\mathrm{Cl}$ & $\mathrm{H}$ & $\mathrm{H}$ & 8.18 & 2.80 \\
$\mathbf{1 6}$ & $\mathrm{H}$ & $\mathrm{H}$ & $\mathrm{COCH}_{3}$ & 7.52 & 2.00 \\
$\mathbf{1 7}$ & $\mathrm{H}$ & $\mathrm{H}$ & $\mathrm{CN}^{2}$ & 7.36 & 1.00 \\
$\mathbf{1 8}$ & $\mathrm{H}$ & $\mathrm{H}$ & $\mathrm{NO}_{2}$ & 6.97 & 1.00 \\
\hline
\end{tabular}

\subsection{1氢键相互作用}

氢键相互作用是氰基与靶标蛋白之间最常见的相 互作用. 氧基在抗癌药物表皮生长因子受体(EGFR)抑 制剂、非核苷类逆转录酶抑制剂(NNRTI)、黄嘌呤氧化 酶(XOD)抑制剂等药物开发中具有重要应用，氧基能够 与靶标蛋白中活性位点的重要氨基酸残基形成氢键相 互作用。

早期研究的 EGFR 抑制剂采用喹唑啉母核如化合物 19, 喹唑啉 3-位的氮原子通过水分子与 Thr830 形成氢 键相互作用, 为了提高 EGFR 抑制剂的活性, 将喹唑啉 母核替换为 3-氰基喹啉环母核(Eq. 2), 氰基中的氮原子 能够直接与 Thr830 形成氢键相互作用, 增强了该类化
合物的药理活性 ${ }^{[12]}$.

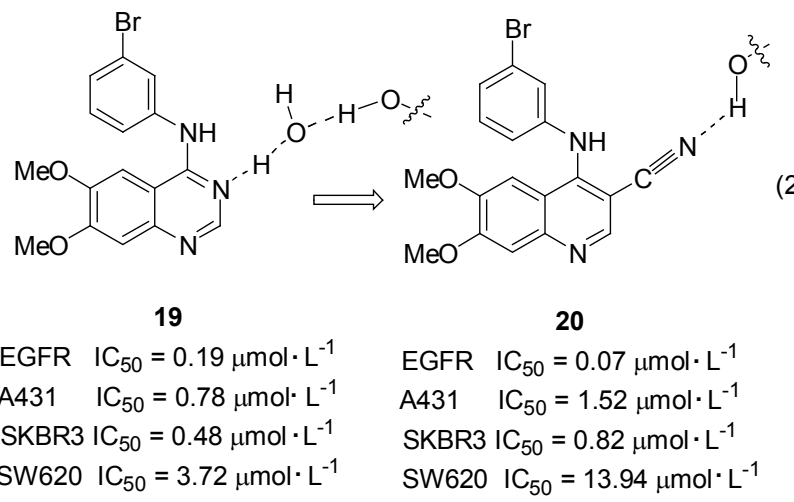

根据此改造策略，惠氏公司设计、合成了氰基喹啉 类 EGFR 抑制剂，包括来那替尼(Neratinib, 21)、培利替 尼(Pelitinib, 22)和伯舒替尼(Bosutinib, 23)(Chart 2). 来 那替尼是由惠氏公司开发的不可逆 EGFR 抑制剂 ( $\mathrm{IC}_{50}$ $=92 \mathrm{nmol} / \mathrm{L})^{[13]}$, 主要用于治疗乳腺癌和非小细胞肺癌, 目前处于临床 III 期研究阶段. 惠氏公司开发的另一个 抗肿瘤药物 $\mathrm{EGFR}$ 抑制剂培利替尼 $\left(\mathrm{IC}_{50}=39 \mathrm{nmol} / \mathrm{L}\right)^{[14]}$ 主要用于实体瘤以及对吉非替尼(Gefitinib, 24)和埃罗替 尼(Erlotinib, 25)具有抗药性的肿瘤治疗，目前处于临床 II 期研究阶段. 伯舒替尼是由惠氏公司开发的强效 Src 激酶 $\left(\mathrm{IC}_{50}=3.8 \mathrm{nmol} / \mathrm{L}\right)$ 和 $\mathrm{Abl}$ 激酶 $\left(\mathrm{IC}_{50}=1.1 \mathrm{nmol} / \mathrm{L}\right)$ 的 双重抑制剂，主要用于治疗慢性粒细胞白血病，目前处 于临床 III 期研究阶段 ${ }^{[15,16]}$.

许多艾滋病治疗药物中也含有芳香氰基这一合成 砌块，其代表药物是非核苷类逆转录酶抑制剂(NNRTI) 的依曲韦林(Etravirine, TMC125-R165335, 26)、达匹韦 林(Dapivirine, 27)和利匹韦林(Rilpivirine, 28)(Chart 3).

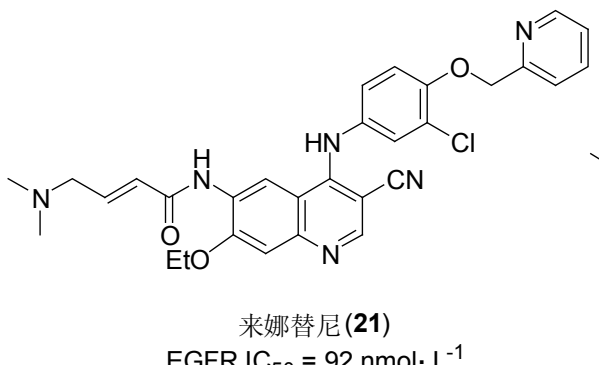
EGFR IC ${ }_{50}=92 \mathrm{nmol} \cdot \mathrm{L}^{-1}$<smiles>COc1cc2ncc(C#N)c(Nc3ccc(F)c(Cl)c3)c2cc1NC(=O)/C=C/CN(C)C</smiles>

培利替尼(22)

EGFR IC $50=39 \mathrm{nmol} \cdot \mathrm{L}^{-1}$<smiles>COc1cc(Nc2c(C#N)cnc3cc(OCCCN4CCN(C)CC4)c(OC)cc23)c(Cl)cc1Cl</smiles>

$\operatorname{Src~IC} C_{50}=3.8 \mathrm{nmol} \cdot \mathrm{L}^{-1}$ Abl IC $\mathrm{C}_{50}=1.1 \mathrm{nmol} \cdot \mathrm{L}^{-1}$<smiles>COc1cc2ncnc(Nc3ccc(F)c(Cl)c3)c2cc1OCCN1CCOCC1</smiles>

吉非替尼(24) EGFR IC $50=1 \mathrm{nmol} \cdot \mathrm{L}^{-1}$

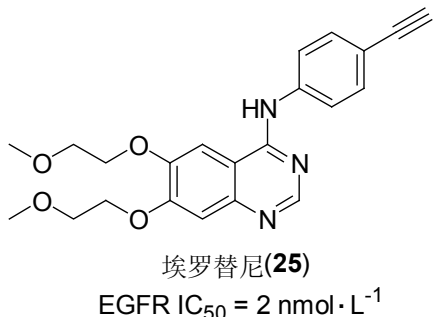

EGFR IC ${ }_{50}=2 \mathrm{nmol} \cdot \mathrm{L}^{-1}$

Chart 2 
<smiles>Cc1cc(C#N)cc(C)c1Oc1nc(Nc2ccc(C#N)cc2)nc(N)c1Br</smiles>

依曲韦林 (26)

$\mathrm{IC}_{50}=38.40 \mathrm{nmol} \cdot \mathrm{L}^{-1}$<smiles>Cc1cc(C)c(Nc2ccnc(Nc3ccc(C#N)cc3)n2)c(C)c1</smiles>

达匹韦林(27)

$\mathrm{IC}_{50}=38.40 \mathrm{nmol} \cdot \mathrm{L}^{-1}$<smiles>Cc1cc(/C=C/C#N)cc(C)c1Nc1ccnc(Nc2ccc(C#N)cc2)n1</smiles>

利匹韦林 (28)

$\mathrm{IC}_{50}<8 \mathrm{nmol} \cdot \mathrm{L}^{-1}$

\section{Chart 3}

其中, 依曲韦林由强生公司研发, 于 2008 年 1 月 18 日 获得美国食品药品监督管理局(FDA)批准上市, 该药物 的显著优点是它与之前开发出的所有非核苷类逆转录 酶抑制剂不具有交叉抗药性，因此，能用于对所有非核 苷类逆转录酶抑制剂产生抗药性的 HIV 病毒感染的治 疗 ${ }^{[17]}$. 晶体复合物表明, 依曲韦林的两个氧基基团较好 地深入到靶标蛋白深处的结合口袋(图 2) ${ }^{[18]}$. 强生公司 开发的另外两个非核苷类逆转录酶抑制剂达匹韦林 ${ }^{[19]}$ 和利匹韦林 ${ }^{[20]}$ 是依曲韦林的类似物, 其中利匹韦林被 称为 “目前发现活性最强的抗艾滋病治疗药物”, 于 2011 年 5 月份获得美国 FDA 批准上市用于艾滋病的治 疗.

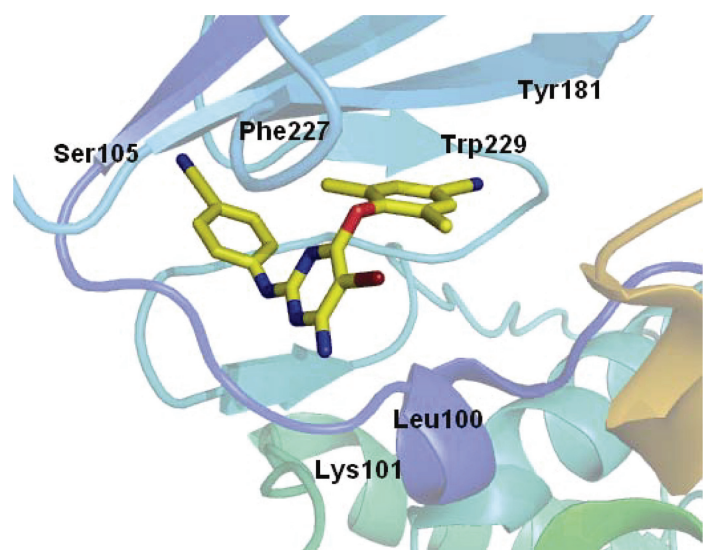

图 2 化合物依曲韦林与逆转录酶的单晶结构示意图

Figrue 2 Crystal structure of binding mode of compound etravirine to reverse transcripatase

非布索坦(Febuxostat, 29)是由武田公司开发的选择 性非嘌呤类黄嘌呤氧化酶抑制剂 $\left(\mathrm{IC}_{50}=114 \mathrm{nmol} / \mathrm{L}\right)$, 用 于治疗痛风性关节炎(Chart 4), 于 2009 年 2 月获得美国 FDA 批准，是近 40 年来首个批准用于治疗高尿酸症的 痛风药物 ${ }^{[21,22]}$. Fuji Yakuhin Kogyo 公司开发的化合物 30 (FYX-051) 的黄嘌呤氧化酶抑制活性更佳 $\left(\mathrm{IC}_{50}=5.3\right.$ $\mathrm{nmol} / \mathrm{L})^{[23]}$. 该化合物与黄嘌呤氧化酶具有更好的结合 能力, 晶体复合物表明, 化合物 FYX-051 的氰基与氨基 酸残基 E802 直接形成氢键相互作用(图 3 ${ }^{[24]}$.

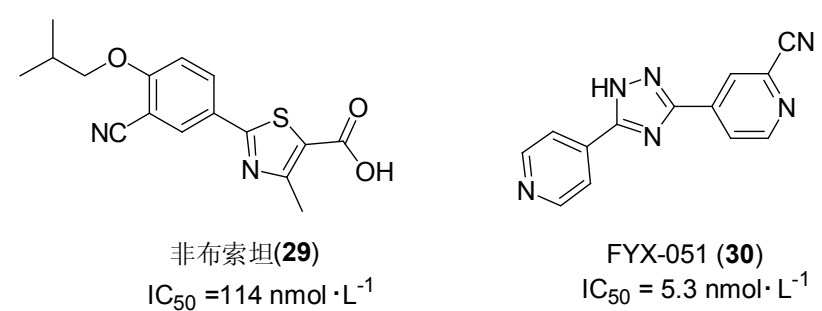

Chart 4

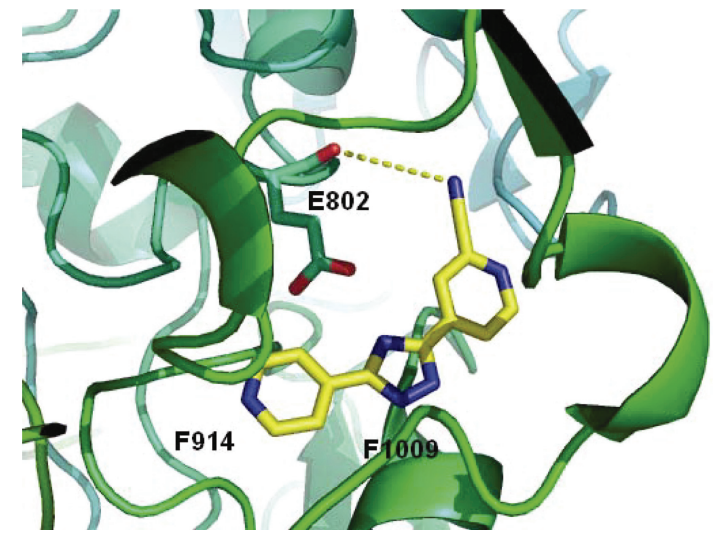

图 3 化合物 FYX-051 与黄嘌呤氧化酶的单晶结构示意图 Figrue 3 Crystal structure of binding mode of compound FYX-051 to xanthine oxidase

\subsection{2 共价相互作用}

组织蛋白酶 $\mathrm{K}$ 抑制剂是一类强效的骨质疏松治疗 药物, 其中化合物 31 具有较强的组织蛋白酶 $\mathrm{K}$ 的抑制 活性 $\left(\mathrm{IC}_{50}<1 \mathrm{nmol} / \mathrm{L}\right)$. 该化合物与组织蛋白酶 $\mathrm{K}$ 的晶体 复合物表明(图 4), $N$-甲基哌嗪环有效地占据了 S3 口袋 并与周围的氨基酸残基 Asp61 以及 Tyr67 形成 $\pi-\pi$ 相互 作用; 环己基有效地占据了 S2 亲酯性口袋; 氧基深入 到蛋白酶的内部，与 Cys25 形成共价相互作用，增强了 该化合物的抑制活性，同时，化合物 31 对组织蛋白酶 L 和组织蛋白酶 $\mathrm{S}$ 的选择性良好 ${ }^{[25]}$.

二肽基肽酶 IV (DPP IV)是丝氨酸蛋白酶家族中的 一员, DPP IV 可以水解胰升血糖素样肽-1 (GLP-1), 降 低其在体内的降糖活性，因此开发有效的 DPP IV 抑制 


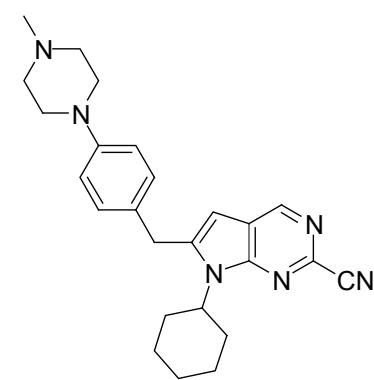

31

Cat $\mathrm{KIC} \mathrm{C}_{50}<1 \mathrm{nmol} \cdot \mathrm{L}^{-1}$

Cat $L I C_{50}=410 \mathrm{nmol} \cdot \mathrm{L}^{-1}$

Cat $\mathrm{S} \mathrm{IC}_{50}=460 \mathrm{nmol} \cdot \mathrm{L}^{-1}$

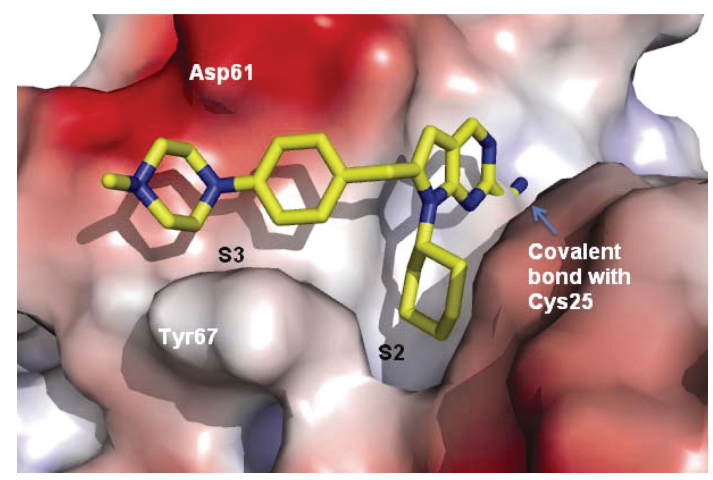

图 4 化合物 31 与组织蛋白酶 $\mathrm{K}$ 的单晶结构示意图

Figrue 4 Crystal structure of binding mode of compound 31 to cathepsin $\mathrm{K}$

剂能切断该水解通路, 成为 II 型糖尿病的有效治疗方 法. Ser630 是该 DPP IV 蛋白酶的关键氨基酸残基, 其中 Ser630 的差基能够与小分子抑制剂中的氧基形成共价 结合, 因此, 许多上市和在研的 DPP IV 抑制剂中都含 有氧基基团，包括 2009 年获得美国 FDA 批准的上市药 物沙格列汀 (Saxagliptin, 1) 和 2007 年获得欧盟批准的 上市药物维格列汀(Vildagliptin, 32) (Chart 5).

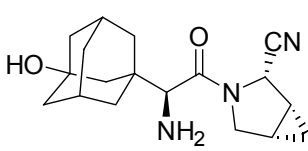

沙格列汀(1) $\mathrm{IC}_{50}=3.37 \mathrm{nmol} \cdot \mathrm{L}^{-1}$

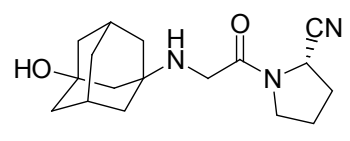

维格列汀(32) $\mathrm{IC}_{50}=3.5 \mathrm{nmol} \cdot \mathrm{L}^{-1}$

\section{Chart 5}

沙格列汀是由施贵宝公司研发的 DPP IV 小分子抑 制剂 $\left(\mathrm{IC}_{50}=3.37 \mathrm{nmol} / \mathrm{L}\right)^{[26]}$, 于 2009 年获得美国 FDA 批 准用于治疗 II 型糖尿病. 沙格列汀与 DPP IV 的活性部 位形成的共价复合物结合模式图如图 5 所示. 氧基碳原 子与 Ser630 中的羟基氧形成共价键. 4,5-亚甲基桥吡咯 环处于 S1 口袋中, 与氨基酸残基 Val711, Val656, Tyr662, Tyr666 和 Tyr547 有范德华相互作用. 金刚烷部
分处于 S2 口袋中. 在 S2 口袋中沙格列汀的羰基氧与 Asn710 的氨基形成氢键; 沙格列汀的 $\mathrm{N}$ 端氨基与氨基 酸残基 Glu205, Glu206 和 Asp663 形成氢键相互作用, 该氢键对沙格列汀的抑制活性具有重要作用; Tyr547 侧 链的羟基分别与金刚烷基的羟基和吡咯环的氰基形成 氢键相互作用.

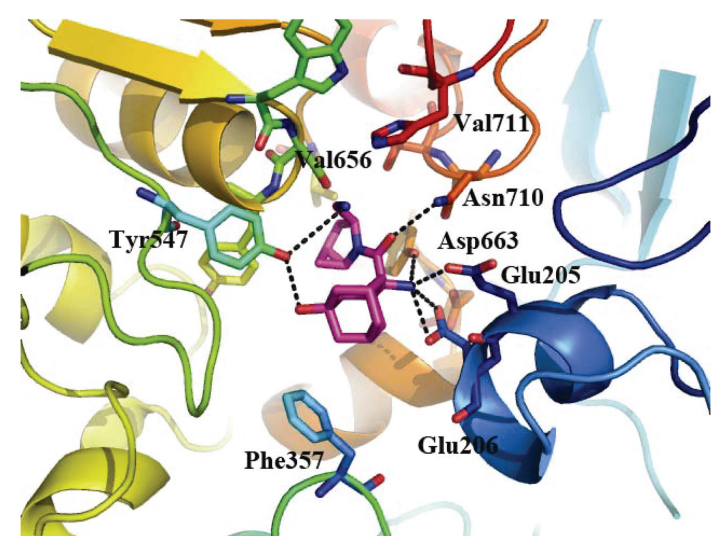

图 5 沙格列汀与二肽基肽酶 IV 的单晶结构示意图

Figrue 5 Crystal structure of binding mode of saxagliptin to DPP IV

维格列汀由诺华公司开发, 是一种具有选择性、竞 争性且可逆的 DPP IV 抑制剂 $\left(\mathrm{IC}_{50}=3.5 \mathrm{nmol} / \mathrm{L}\right)^{[27]}$, 已 于 2007 年获得欧盟批准在欧洲上市, 用于 II 型糖尿病 的治疗. 健康人体药动学研究表明, 口服维格列汀吸收 迅速，生物利用度约为 $85 \%$, 达峰时间为给药后 $1 \sim 2 \mathrm{~h}$, 蛋白结合率低( $4 \% \sim 17 \%)$, 血浆半衰期为 $1.5 \sim 4.5 \mathrm{~h}$, 但 对体内血浆中 DPP IV 的抑制作用则长达 $10 \mathrm{~h}^{[28,29]}$.

\subsection{3 偶极相互作用}

由于氧基具有较强的吸电子特性，因此氰基能够与 氨基酸残基和金属离子产生非特异性偶极相互作用.

维拉帕米 (Verapamil, 33) 是钙离子通道拮抗剂 $\left(\mathrm{IC}_{50}=0.47 \mu \mathrm{mol} / \mathrm{L}\right)^{[30]}$, 主要用于治疗心律失常和心绞 痛(Chart 6). 维拉帕米可以降低血压, 进而减少心输出 量, 同时增加心脏中的血液和氧气, 减少胸痛. 戈洛帕 米(Gallopamil, 34)是维拉帕米的甲氧基取代衍生物, 比 维拉帕米的活性增加近 10 倍 $\left(\mathrm{IC}_{50}=1.76 \mu \mathrm{mol} / \mathrm{L}\right)$, 分子 模拟表明氰基是活性必需基团，与钻离子形成偶极相互 作用(图 6) ${ }^{[31]}$.

西洛司特(Cilomilast, 35)是由葛兰素史克公司开发 的选择性磷酸二酯酶 4 抑制剂, 用于治疗炎症和哮喘 $\left(\mathrm{IC}_{50}=95 \mathrm{nmol} / \mathrm{L}\right)^{[32]}$, 目前处于临床 III 期研究阶段. 晶 体复合物结构表明, 氧基与周围的氨基酸残基 M347 和 L393 形成偶极相互作用(图 7) ${ }^{[33]}$. 通过结构改造, 优化 该类化合物的活性, 减少恶心、腹泻和头痛等副作用. 
<smiles>COc1ccc(CCN(C)CCCC(C#N)(c2ccc(OC)c(OC)c2)C(C)C)cc1OC</smiles>

维拉帕米(33)

$\mathrm{IC}_{50}=14.0 \mu \mathrm{mol} \cdot \mathrm{L}^{-1}$

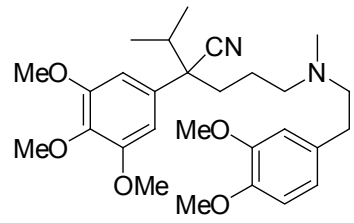

戈洛帕米(34)

$\mathrm{IC}_{50}=1.76 \mu \mathrm{mol} \cdot \mathrm{L}^{-1}$
Chart 6

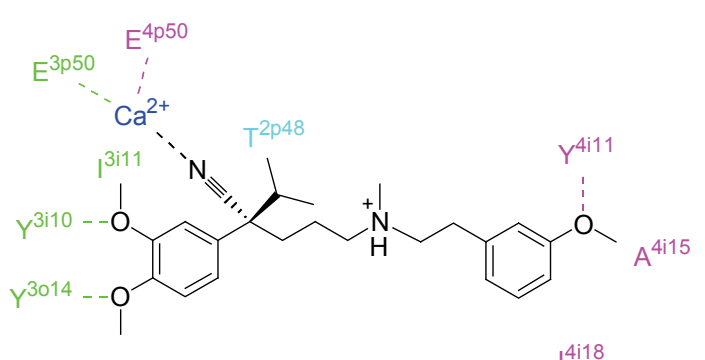

$4^{4 i 18}$

图 6 含氧基的钲离子通道拮抗剂剂的分子模拟意图

Figrue 6 Molecular modeling of binding mode of compound containing nitrile group to calcium channel<smiles>COc1ccc(C2(C)CCC(C(=O)O)CC2)cc1OC1CCCC1</smiles>

西洛司特(35)

$\mathrm{IC}_{50}=95 \mathrm{nmol} \cdot \mathrm{L}^{-1}$

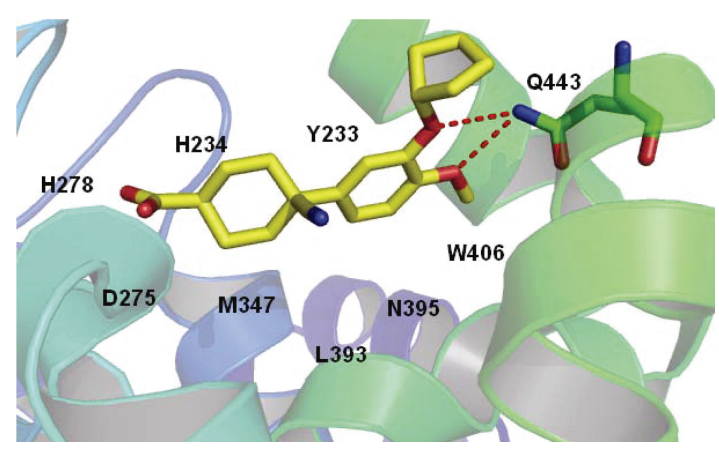

图 7 西洛司特与磷酸二酯酶 4 的单晶结构示意图

Figrue 7 Crystal structure of binding mode of cilomilast to PDE4

\section{$2.1 .4 \pi-\pi$ 相互作用}

施贵宝公司开发的雷夫康唑(Ravuconazole, 36)是 口服有效的新型强效广谱抗真菌剂, 安全性良好, 目前 处于临床 II 期研究阶段 ${ }^{[34]}$. 雷夫康唑抗白色念珠菌、光 滑念珠菌、新型隐球菌和熏烟色曲霉菌的活性强于依曲 康唑(Itraconazole), 对鼠全身及肺曲霉菌病的活性强于 依曲康唑. 雷夫康唑与甾醇 $14 \alpha$-去甲基化酶的对接结果 表明, 雷夫康唑中的噻唑环与氨基酸残基 Tyr131, Leu134 以及 Ile389 形成相互作用, 其中 4-氭基苯基与氨
基酸残基 Phe240, Met528 以及 Ile529 形成疏水相互作 用, 氰基取代的苯环结构与氨基酸残基 His 133 形成 $\pi-\pi$ 相互作用。<smiles>CC(c1nc(-c2ccc(C#N)cc2)cs1)C(O)(c1ccccc1)c1ccc(F)cc1F</smiles>

Chart 7

\section{2 作为生物电子等排体}

\subsection{1 羰基的生物电子等排体}

在许多非甾体芳香酶抑制剂的研发过程中，药物化 学研究人员采用氭基作为羰基的电子等排体，一方面提 高了小分子的靶标选择性, 减少了与其它相关甾体类受 体的相互作用; 另一方面可以改善药物小分子的口服生 物利用度. 4-氧基取代苯环中的氧基氮原子与孕酮 3-位 的羰基占据了相同的靶标蛋白结合位点, 能够与关键氨 基酸残基形成氢键相互作用(Chart 8).
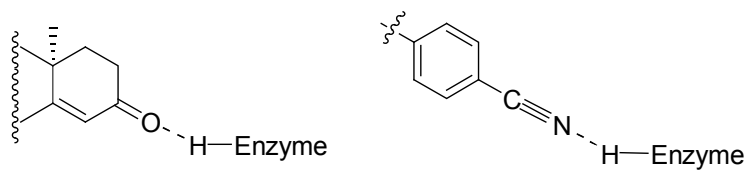

\section{Chart 8}

氯基是羰基的经典生物电子等排体，与羰基相比， 氰基具有非特异性的极性相互作用. 通常在芳香环的对 位引入氰基, 能够提高芳香环的极化作用, 使得芳香体 系更加稳定而不易发生氧化代谢, 提高了化合物的药物 代谢稳定性. 许多芳香酶抑制剂在苯环的对位引入氧基 取代，苯环 4-位的氰基基团模拟了雌激素甾体类化合物 的羰基，与周围的氢键受体形成氢键相互作用，氰基的 引入有效地提高了该类化合物的芳香酶抑制活性. 诺华 公司开发的芳香酶抑制剂法曲唑(Fadrozole，37)是第一 个用于治疗乳腺癌的非甾体芳香酶抑制剂 $\left(\mathrm{IC}_{50}=30\right.$ $\mathrm{nmol} / \mathrm{L})\left(\right.$ Chart 9) ${ }^{[35]}$ ，构效关系表明，苯环上 4-位为吸 电子的溴原子取代和氧基取代时活性最优. 诺华公司后 续开发的来曲唑(Letrozole, 38)主要用于辅助治疗荷尔 蒙引起的乳腺癌 $\left(\mathrm{IC}_{50}=0.29 \mathrm{nmol} / \mathrm{L}\right)^{[36]}$. Hormos 公司研 发的芬罗唑(Finrozole, 39) ${ }^{[37]}$ 同样含有氭基苯基这一结 构片段，该化合物是芳香酶和醛固酮的双重抑制剂，目 前处于临床 II 期研究阶段. 芬罗唑中的氧基就是模仿甾 体化合物中的羰基与氢键受体形成相互作用. 单晶结构 表明(图 8), 4-氧基与 His93L 和 Phe94L 形成氢键相互作 
用 ${ }^{[38]}$.

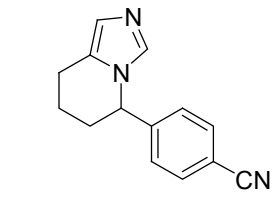

法曲唑(37)

$\mathrm{IC}_{50}=30 \mathrm{nmol} \cdot \mathrm{L}^{-1}$<smiles>N#Cc1ccc(C([C@H](O)Cc2ccc(F)cc2)n2cncn2)cc1</smiles>

芬罗唑(39)

$\mathrm{IC}_{50}=0.29 \mathrm{nmol} \cdot \mathrm{L}^{-1}$

Chart 9

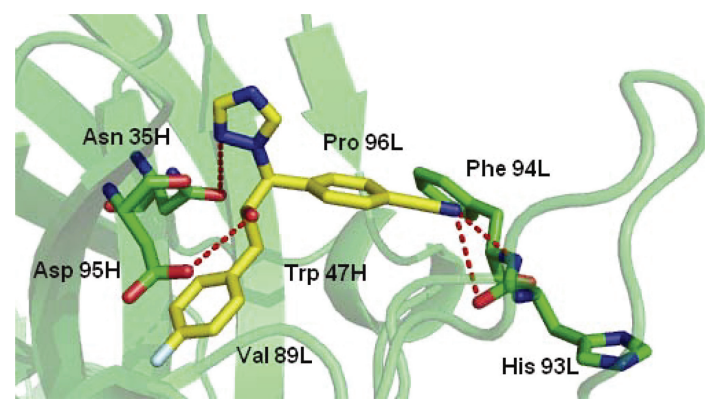

图 8 芬罗唑与芳香酶的单晶结构示意图

Figrue 8 Crystal structure of binding mode of finrozole to aromatase

由阿斯利康公司开发的雄激素受体拮抗剂比卡鲁 胺(Bicalutamide, 40)于 1995 年获得美国 FDA 批准, 是前 列腺癌治疗的代表药物 ${ }^{[39]}$. 比卡鲁胺口服生物利用度 良好，达到了 $90 \%$ ，同时对其它甾体受体无拮抗作用， 具有良好的靶标蛋白选择性. 比卡鲁胺中的氰基与氨基 酸残基 M745, R752 和 Q711 形成氢键相互作用(图 9), 增强了该化合物与靶标蛋白的相互作用, 提高了化合物 的拮抗活性 $\left(\mathrm{IC}_{50}=190 \mathrm{nmol} / \mathrm{L}\right)^{[40]}$.

芳氧基作为甾体类化合物羰基的生物电子等排体 的另一个例子是黄体酮(Progesterone, 41) 与他那特罗 (Tanaproget, 42). 惠氏公司研发的他那罗特是一种新型 组织选择性非甾体类㭆激素受体激动剂 $\left(\mathrm{EC}_{50}=0.15\right.$ $\mathrm{nmol} / \mathrm{L}$ ), 目前处于临床 II 期研究阶段 ${ }^{[41]}$. 单晶结构表 明，他那罗特中的芳氧基与关键氨基酸残基 $\mathrm{G} \ln 725$, $\operatorname{Arg} 766$ 以及水分子形成氢键相互作用(图 10) ${ }^{[42,43]}$.

\subsection{2 卤素的生物电子等排体}

氧基能够模拟卤素产生的极性相互作用, 因而成为 卤素的生物电子等排体. 氧基的体积小于溴原子和碘原 子, 氧基中的氮原子能够更好地与周围的氨基酸残基形<smiles>N#Cc1ccc(NC(=O)C2(O)CS(=O)(=O)O2)cc1C(F)(F)F</smiles>

比卡鲁胺(40)

$\mathrm{IC}_{50}=190 \mathrm{nmol} \cdot \mathrm{L}^{-1}$ $F=90 \%$

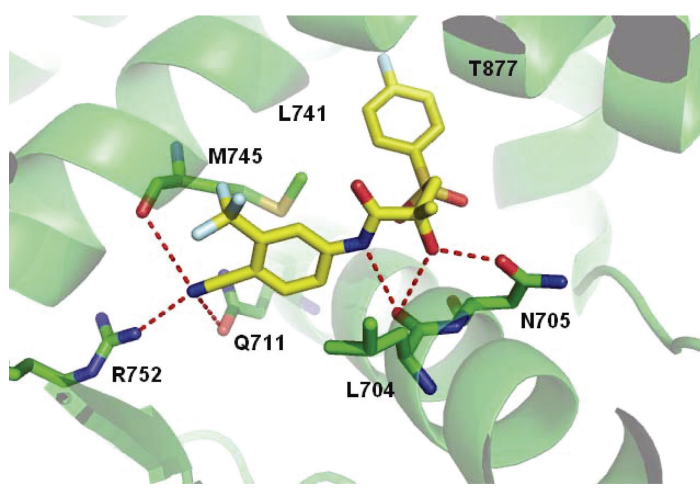

图 9 比卡鲁胺与雄激素受体的单晶结构示意图

Figrue 9 Crystal structure of binding mode of bicalutamide to androgen receptor

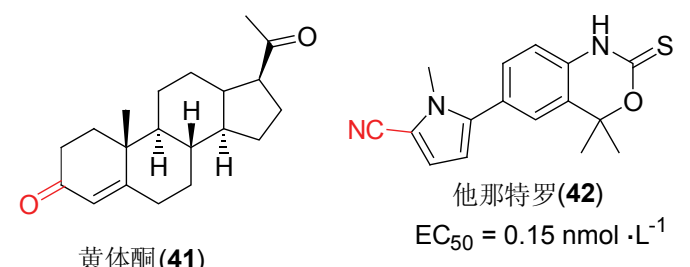

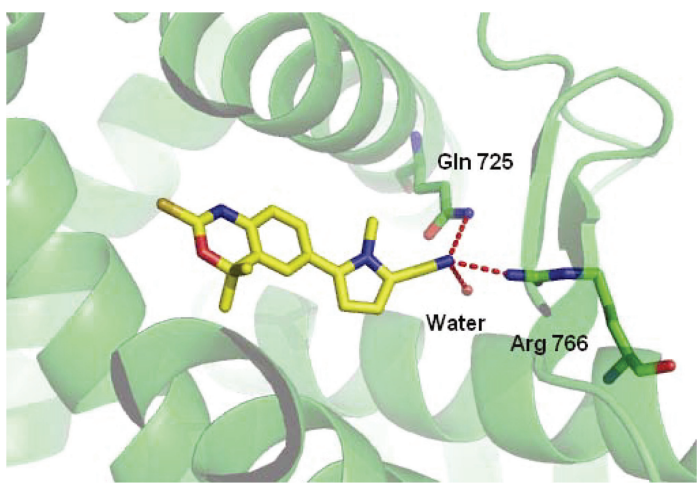

图 10 他那特罗与黄体酮受体的单晶结构示意图

Figrue 10 Crystal structure of binding mode of tanaproget to progesterone receptor

成相互作用.

磷酸二酯酶抑制剂米力农(Milrinone, 43) 是治疗心 力衰竭的首选药物 (Chart 10$)^{[44]}$. 米力农的结构与甲状 腺素类似，同时刺激心肌膜具有与荷尔蒙类似的功能. 奥普力农(Olprinone, 44) 是米力农的类似物, 通过增强 心肌收缩能力用于治疗心力衰竭的血管舒张剂 ${ }^{[45]}$. 奥 普力农与甲状腺素运载蛋白的晶体复合物表明, 氧基吡 啶酮部分位于荷尔蒙的结合口袋 ${ }^{[46]}$. 氧基占据了与甲 
状腺素中碘原子的结合口袋，并与氨基酸残基形成了紧 密结合. 将氰基替换为氨基, 活性保持, 但是不能与周 围的氨基酸残基形成较好的相互作用. 虽然溴原子也能 较好地与氨基酸残基进行作用深入结合口袋, 但是氰基 的疏水性更强. 氧基是碘原子和溴原子的非经典生物电 子等排体, 是先导化合物结构优化中所采取的重要研究 策略.<smiles>Cc1[nH]c(=O)c(C#N)cc1-c1ccncc1</smiles>

米力农(43)

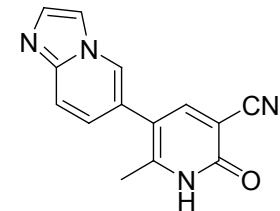

奥普力农 $(44)$
Chart 10

维拉佐酥(Vilazodone, 54) 是由默克公司开发的选择 性五差色胺重摄取抑制剂 $\left(\mathrm{IC}_{50}=0.3 \mathrm{nmol} / \mathrm{L}\right)$, 于 2011 年 1 月 21 日获得美国食品药品监督管理局(FDA)批准上市, 用于成人抑郁症的治疗. 通过对一系列类似物的笁选, 最终发现氟原子和氰基取代的化合物活性最强，将 $\mathrm{X}$ 基 团替换为氟原子和氰基基团活性相当(表 2), 表明氰基 是氟原子的生物电子等排体 ${ }^{[47]}$.

表 2 氟取代和氧基取代对吲哚类化合物活性的影响 [( $\mathrm{IC}_{50} \pm$ $\left.\mathrm{SEM}) /\left(\mathrm{nmol} \cdot \mathrm{L}^{-1}\right)\right]$

Table 2 Effect of fluorine and nitrile substitution on activities of indole compounds $\left[\left(\mathrm{IC}_{50} \pm \mathrm{SEM}\right) /\left(\mathrm{nmol} \cdot \mathrm{L}^{-1}\right)\right]$

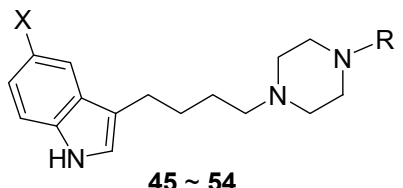

$$
45 \sim 54
$$

\begin{tabular}{|c|c|c|c|c|c|}
\hline Compd. & $\mathrm{R}$ & $X$ & $5-\mathrm{HT}_{1 \mathrm{~A}}$ & RUI & $\mathrm{D}_{2}$ \\
\hline 45 & & $\mathrm{~F}$ & $40 \pm 15$ & $2.0 \pm 0.6$ & $>100$ \\
\hline 46 & & $\mathrm{CN}$ & $1.1 \pm 0.2$ & $1.3 \pm 0.9$ & $255 \pm 45$ \\
\hline 47 & & $\mathrm{~F}$ & $4 \pm 2$ & $1 \pm 0.5$ & $<100$ \\
\hline 48 & & $\mathrm{CN}$ & $0.7 \pm 0.4$ & $0.6 \pm 0.06$ & $4.9 \pm 1.2$ \\
\hline 49 & & $\mathrm{~F}$ & $5 \pm 2$ & $3 \pm 1$ & $>100$ \\
\hline 50 & & $\mathrm{CN}$ & $0.4 \pm 0.3$ & $1 \pm 0.7$ & $>630$ \\
\hline 51 & & $\mathrm{~F}$ & $0.7 \pm 0.2$ & $0.9 \pm 0.3$ & $>100$ \\
\hline 52 & & $\mathrm{CN}$ & $0.1 \pm 0.06$ & $0.7 \pm 0.5$ & $740 \pm 232$ \\
\hline 53 & & $\mathrm{~F}$ & $3.0 \pm 0.9$ & $0.3 \pm 0.2$ & $>100$ \\
\hline 54 & & $\mathrm{CN}$ & $0.3 \pm 0.06$ & $0.5 \pm 0.4$ & $666 \pm 75$ \\
\hline
\end{tabular}

\section{3 药物的药代性质}

\section{1 提高代谢稳定性}

氧基能够改善小分子化合物的药代动力学性质. 计 算机辅助药物设计以及经验规则(例如 Lipinski 五规则) 是基于结构药物设计的重要指导原则. 当发现了一个具 有较强药理活性的小分子之后，改善药物的药代动力学 特性就成为化合物开发成为药物的重要决定因素. 先导 化合物优化通常会增加化合物的复杂性和脂溶性，而引 入氰基基团能够有效地降低脂溶性，例如：用氰基替换 氢原子能够显著地降低 $c \log P$, 而将卤素或甲基替换为 氧基, $c \log P / \log D$ 的比值也将减少.

通常来说, 提高 II 相代谢稳定性的结构修饰主要包 括以下三种策略：(1)引入吸电子基团和空间位阻; (2)将 酚差基改造为环脲或者硫脲基团; (3)将酚羟基转变为前 药. 在酚羟基类胰高血糖素受体拮抗剂的邻位引入氧基 基团，能够有效减少该类化合物与葡萄糖醛酸的结合 (表 3) ${ }^{[48]}$. 将该类化合物 $\mathrm{R}^{1}$ 基团从氯原子替换为氰基, 使得该类化合物与葡萄糖醛酸结合活性降低，进而减慢 其代谢清除速率，其中化合物 60 比化合物 59 的代谢清 除速率降低了 4 倍, 增强了化合物的代谢稳定性，延长 了化合物在体内的作用时间.

表 3 氰基取代对代谢稳定性的影响

Table 3 Effect of nitrile substitution on metabolic stability<smiles>[R]c1cc(C(=O)N/N=C/c2ccc([R])c3ccccc23)ccc1O</smiles>

\begin{tabular}{ccccc}
\hline $\begin{array}{c}\text { 化合 } \\
\text { 物 }\end{array}$ & $\mathrm{R}^{1}$ & $\mathrm{R}^{2}$ & $\begin{array}{c}\mathrm{hGluR} \text { 亲和性/ } \\
\left(\mathrm{nmol} \cdot \mathrm{L}^{-1}\right)\end{array}$ & $\begin{array}{c}\text { 代谢清除率/ } \\
\left(\mathrm{pmol} \cdot \mathrm{min}^{-1} \cdot \mathrm{mg}^{-1}\right)\end{array}$ \\
\hline $\mathbf{5 5}$ & $\mathrm{Cl}$ & $\mathrm{CH}_{2} \mathrm{OH}$ & 41.1 & 75 \\
$\mathbf{5 6}$ & $\mathrm{F}$ & $\mathrm{CH}_{2} \mathrm{OH}$ & 29.4 & 89 \\
$\mathbf{5 7}$ & $2,3-\mathrm{Cl}_{2}$ & $\mathrm{CH}_{2} \mathrm{OH}$ & 53.8 & 103 \\
$\mathbf{5 8}$ & $\mathrm{CN}$ & $\mathrm{CH}_{2} \mathrm{OH}$ & 29.8 & 37 \\
$\mathbf{5 9}$ & $\mathrm{Cl}$ & $\mathrm{O}$ & 8.3 & 267 \\
& & & 11.6 & 65 \\
\hline
\end{tabular}

\section{2 延长在体内的作用时间}

化合物 61 (Capravirine) 的结构改造过程是延长药物 在体内的作用时间, 改善药代动力学特性的最佳例证

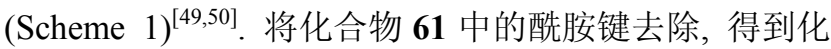
合物 62, 其逆转录酶的抑制活性减低 $\left(\mathrm{IC}_{50}=0.56\right.$ $\mu \mathrm{mol} / \mathrm{L})$, 脂溶性增加 $(c \log P=4.0)$. 采用氭基作为氯原 子的生物电子等排体，将苯环上的氯原子替换为㲵基， 
其逆转录酶的抑制活性提高 $\left(\mathrm{IC}_{50}=0.35 \mu \mathrm{mol} / \mathrm{L}\right)$, 脂溶 性降低了 1 个单位 $(c \log P=2.7)$, 同时增强了脂溶性配 体作用(lipophilic ligand efficiency, LLE) ${ }^{[51]}$. 在化合物 63 的结构基础上再引入一个氰基, 得到了化合物(64, Lersivirine), 该化合物的生物活性与化合物 61 的抑制活性 相当 $\left(\mathrm{IC}_{50}=0.119 \mu \mathrm{mol} / \mathrm{L}\right)$, 分子量减少了 141 , 脂溶性 降低 $(c \log P=2.1)$, 同时增加了在人类肝线粒体的半衰 期, 从化合物 61 的 $7.5 \mathrm{~min}$ 半衰期延长到 $73 \mathrm{~min}$, 提高 了近 10 倍.
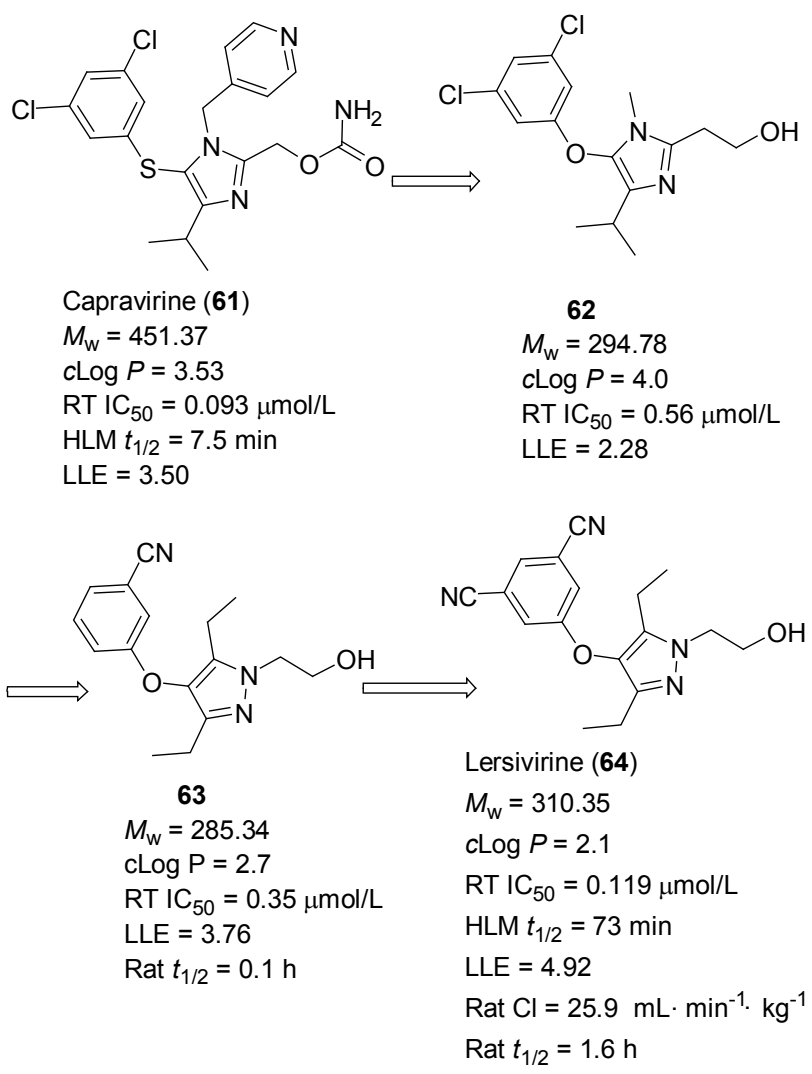

Scheme 1

\section{4 结论}

结构丰富、种类多样的含氰基药物已经广泛用于临 床治疗. 通过对含有氭基的上市药物以及临床候选药物 的调研, 发现氧基在药物的生理功能中发挥着不同的作 用. 在许多药物中, 㲵基对周围的官能团产生极化作用, 增加其电子云密度; 在其它药物中, 氧基则作为重要的 分子识别基团, 有效地与靶标蛋白结合, 产生生理活性. 本综述总结了氰基与多种靶标的相互作用, 归纳了含氧 基药物和候选药物的结构相似性以及在不同生物模型 中的重要作用, 深入理解了氰基在先导化合物优化过程 中所起到的重要作用.

\section{References}

[1] Fleming, F. F.; Yao, L. H.; Ravikumar, P. C.; Funk, L.; Shook, B. C. J. Med. Chem. 2010, 53, 7902.

[2] Augeri, D. J.; Robl, J. A.; Betebenner, D. A.; Magnin, D. R.; Khanna, A.; Robertson, J. G.; Wang, A.; Simpkins, L. M.; Taunk, P.; Huang, Q.; Han, S. P.; Abboa-Offei, B.; Cap, M.; Xin, L.; Tao, L.; Tozzo, E.; Welzel, G. E.; Egan, D. M.; Marcinkeviciene, J.; Chang, S. Y.; Biller, S. A.; Kirby, M. S.; Parker, R. A.; Hamann, L. G. J. Med. Chem. 2005, 48, 5025.

[3] El-Kemary, M.; Organero, J. A.; Douhal, A. J. Med. Chem. 2006, 49, 3086.

[4] Teodori, E.; Dei, S.; Garnier-Suillerot, A.; Gualtieri, F.; Manetti, D.; Martelli, C.; Romanelli, M. N.; Scapecchi, S.; Sudwan, P.; Salerno, M. J. Med. Chem. 2005, 48, 7426.

[5] Pascual, E.; Sivera, F.; Yasothan, U.; Kirkpatrick, P. Nat. Rev. Drug Discovery 2009, 8, 191.

[6] Hughes, C. A.; Robinson, L.; Tseng, A.; MacArthur, R. D. Expert Opin. Pharmacother. 2009, 10, 2445.

[7] Fradet, Y. Expert Rev. Anticancer Ther. 2004, 4, 37.

[8] Bell, I. M. J. Med. Chem. 2004, 47, 1869.

[9] Reid, T. S.; Besse, L. S. Biochemistry 2004, 43, 6877.

[10] Hunt, J. T.; Ding, C. Z.; Batorsky, R.; Bednarz, M.; Bhide, R.; Cho, Y.; Chong, S.; Chao, S.; Gullo-Brown, J.; Guo, P.; Kim, S. K.; Lee, F. Y. F.; Leftheris, K.; Miller, A.; Mitt, T.; Patel, M.; Penhallow, B. A.; Ricca, C.; Rose, W. C.; Schmidt, R. C.; Slusarchyk, W. A.; Vite, G.; Manne, V. J. Med. Chem. 2000, 43, 3587.

[11] Miller, G. H.; Doukas, P. H.; Seydel, J. K. J. Med. Chem. 1972, 15, 700 .

[12] Wissner, A.; Berger, D. M.; Boschelli, D. H.; Floyd, J. B.; Greenberger, L. M.; Gruber, B. C.; Johnson, B. D.; Mamuya, N.; Nilakantan, R.; Reich, M. F.; Shen, R.; Tsou, H.; Upelacis, E.; Wang, Y. F.; Wu, B.; Ye, F.; Zhang, N. J. Med. Chem. 2000, 43, 3244.

[13] Rabindran S. K.; Discafani, C. M.; Rosfjord, E. C.; Baxter, M.; Floyd, M. B.; Golas, J.; Hallett, W. A.; Johnson, B. D.; Nilakantan, R.; Overbeek, E.; Reich, M. F.; Shen, R.; Shi, X.; Tsou, H. R.; Wang, Y. F.; Wissner, A. Cancer Res. 2004, 64, 3958.

[14] Lee, Y. J.; Kim, J. H.; Kim, S. K.; Ha, S. J.; Mok, T. S.; Mitsudomi, T.; Cho, B. C. Lung Cancer 2011, 72, 9.

[15] Morphy, R. J. Med. Chem. 2010, 53, 1413.

[16] Gunby, R. H.; Ahmed, S.; Sottocornola, R.; Gasser, M.; Redaelli, S.; Mologni, L.; Tartari, C. J.; Belloni, V.; Gambacorti-Passerini, C.; Scapozza, L. J. Med. Chem. 2006, 49, 5759.

[17] Hughes, C. A.; Robinson, L.; Tseng, A.; MacArthur, R. D. Expert Opin. Pharmacother. 2009, 10, 2445.

[18] Das, K.; Clark, A. D. Jr.; Lewi, P. J.; Heeres, J.; De Jonge, M. R.; Koymans, L. M. H.; Vinkers, H. M.; Daeyaert, F.; Ludovici, D. W.; Kukla, M. J.; De Corte, B.; Kavash, R. W.; Ho, C. Y.; Ye, H.; Lichtenstein, M. A.; Andries, K.; Pauwels, R.; de Béthune, M. P.; Boyer, P. L.; Clark, P.; Hughes, S. H.; Janssen, P. A. J.; Arnold, E. J. Med. Chem. 2004, 47, 2550.

[19] Nuttall, J. P.; Thake, D. C.; Lewis, M. G.; Ferkany, J. W.; Romano, J. W.; Mitchnick, M. A. Antimicrob. Agents Chemother. 2008, 52, 909.

[20] De Clercq, E. Expert Opin. Emerging Drugs 2005, 10, 241.

[21] Pascual, E.; Sivera, F.; Yasothan, U.; Kirkpatrick, P. Nat. Rev. Drug Discovery 2009, 8, 191.

[22] Okamoto, K.; Eger, B. T.; Nishino, T.; Kondo, S.; Pai, E. F.; Nishino, T. J. Biol. Chem. 2003, 278, 1848.

[23] Sato, T.; Ashizawa, N.; Matsumoto, K.; Iwanaga, T.; Nakamura, H.; Inoue, T.; Nagata, O. Bioorg. Med. Chem. Lett. 2009, 19, 6225. 
[24] Okamoto, K.; Matsumoto, K.; Hille, R.; Eger, B. T.; Pai, E. F.; Nishino, T. Proc. Natl. Acad. Sci. U. S. A. 2004, 101, 7931.

[25] Teno, N.; Miyake, T.; Ehara, T.; Irie, O.; Sakaki, J.; Ohmori, O.; Gunji, H.; Matsuura, N.; Masuya, K.; Hitomi, Y.; Nonomura, K.; Horiuchi, M.; Gohda, K.; Iwasaki, A.; Umemura, I.; Tada, S.; Kometani, M.; Iwasaki, G.; Cowan-Jacob, S. W.; Missbach, M.; Lattmann, R.; Betschart, C. Bioorg. Med. Chem. Lett. 2007, 17, 6096.

[26] Augeri, D. J.; Robl, J. A.; Betebenner, D. A.; Magnin, D. R.; Khanna, A.; Robertson, J. G.; Wang, A.; Simpkins, L. M.; Taunk, P.; Huang, Q.; Han, S.-P.; Abboa-Offei, B.; Cap, M.; Xin, L.; Tao, L.; Tozzo, E.; Welzel, G. E.; Egan, D. M.; Marcinkeviciene, J.; Chang, S. Y.; Biller, S. A.; Kirby, M. S.; Parker, R. A.; Hamann, L. G. J. Med. Chem. 2005, 48, 5025 .

[27] Villhauer, E. B.; Brinkman, J. A.; Naderi, G. B.; Burkey, B. F.; Dunning, B. E.; Prasad, K.; Mangold, B. L.; Russel, M. E.; Hughes, T. E. J. Med. Chem. 2003, 46, 2774.

[28] Ristic, S.; Byiers, S.; Foley, J.; Holmes, D. Diabetes, Obes. Metab. 2005, 6, 692 .

[29] He, H.; Tran, P.; Yin, H.; Smith, H.; Batard, Y.; Wang, L.; Einolf, H.; Gu, H.; Mangold, J. B.; Fischer, V.; Howard, D. Drug Metab. Dispos. 2009, 37, 536.

[30] Cooper-DeHoff, R. M.; Handberg, E. M.; Mancia, G.; Zhou, Q.; Champion, A.; Legler, U. F.; Pepine, C. J. Expert Rev. Cardiovasc. Ther. 2009, 7, 1329.

[31] Cheng, R. C. K.; Tikhonov, D. B.; Zhorov, B. S. J. Biol. Chem. 2009, 284, 28332.

[32] Christensen, S.; Guider, A.; Forster, C. J.; Gleason, J. G.; Bender, P. E.; Karpinski, J. M.; Dewolf, W. E.; Barnette, M. S.; Underwood, D. C.; Griswold, D. E.; Cieslinski, L. B.; Burman, M.; Bochnowicz, S.; Osborn, R. R.; Manning, C. D.; Grous, M.; Hillegas, L. M.; Bartus, J. O.; Ryan, M. D.; Eggleston, D. S.; Haltiwanger, R. C.; Torphy, T. J. J. Med. Chem. 1998, 41, 821.

[33] Card, G. L.; England, B. P.; Suzuki, Y.; Fong, D.; Powell, B.; Lee, B.; Luu, C.; Tabrizizad, M.; Gillette, S.; Ibrahim, P. N.; Artis, D. R.; Bollag, G.; Milburn, M. V.; Kim, S.-H.; Schlessinger, J.; Zhang, K. Y. J. Structure 2004, 12, 2233.

[34] Sheng, C.; Miao, Z.; Ji, H.; Yao, J.; Wang, W.; Che, X.; Dong, G.; Lv, J.; Guo, W.; Zhang, W. Antimicrob. Agents Chemother. 2009, $53,3487$.

[35] Browne, L. J.; Gude, C.; Rodriguez, H.; Steele, R. E.; Bhatnager, A. J. Med. Chem. 1991, 34, 725 .
[36] Bhatnagar, A. S. Breast Cancer Res. Treat. 2007, 105, 7.

[37] Parkkinen, T.; Nevanen, T. K.; Koivula, A.; Rouvinen, J. J. Med. Chem. 2000, 43, 581.

[38] Parkkinen, T.; Nevanen, T. K.; Koivula, A.; Rouvinen, J. J. Mol. Biol. 2006, 357, 471.

[39] Gao, W.; Bohl, C. E.; Dalton, J. T. Chem. Rev. 2005, 105, 3352.

[40] Bohl, C. E.; Gao, W.; Miller, D. D.; Bell, C. E.; Dalton, J. T. Proc. Natl. Acad. Sci. U. S. A. 2005, 102, 6201.

[41] Kern, J. C.; Terefenko, E.; Trybulski, E.; Berrodin, T. J.; Cohen, J.; Winneker, R. C.; Yudt, M. R.; Zhang, Z.; Zhu, Y.; Zhang, P. Bioorg. Med. Chem. Lett. 2009, 19, 6666.

[42] Zhou, H. B.; Lee, J. H.; Mayne, C. G.; Carlson, K. E.; Katzenellenbogen, J. A. J. Med. Chem. 2010, 53, 3349.

[43] Fensome, A.; Bender, R.; Chopra, R.; Cohen, J.; Collins, M. A.; Hudak, V.; Malakian, K.; Lockhead, S.; Olland, A.; Svenson, K.; Terefenko, E. A.; Unwalla, R. J.; Wilhelm, J. M.; Wolfrom, S.; Zhu, Y.; Zhang, Z.; Zhang, P.; Winneker, R. C.; Wrobel, J. J. Med. Chem. 2005, 48, 5092.

[44] Hannon, J. D.; Housmans, P. R. Adv. Cardiovasc. Pharmacol. 2008, 1 .

[45] Mizushige, K.; Ueda, T.; Yukiiri, K.; Suzuki, H. Cardiovasc. Drug. Rev. 2002, 20, 163.

[46] Wojtczak, A.; Luft, J. R.; Cody, V. J. Biol. Chem. 1993, 268, 6202.

[47] Heinirich, T.; Böttcher, H.; Gericke, R.; Bartoszyk, G. D.; Anzali, S.; Seyfried, C. A.; Greiner, H. E.; van Amsterdam, C. J. Med. Chem. 2004, 47, 4684.

[48] Madsen, P.; Ling, A.; Plewe, M.; Sams, C. K.; Knudsen, L. B.; Sidelmann, U. G.; Ynddal, L.; Brand, C. L.; Andersen, B.; Murphy, D.; Teng, M.; Truesdale, L.; Kiel, D.; May, J.; Kuki, A.; Shi, S.; Johnson, M. D.; Teston, K. A.; Feng, J.; Lakis, J.; Anderes, K.; Gregor, V.; Lau, J. J. Med. Chem. 2002, 45, 5755.

[49] Mowbray, C. E.; Corbau, R.; Hawes, M.; Jones, L. H.; Mills, J. E.; Perros, M.; Selby, M. D.; Stupple, P. A.; Webster, R.; Wood, A. Bioorg. Med. Chem. Lett. 2009, 19, 5603.

[50] Mowbray, C. E; Burt, C.; Corbau, R.; Gayton, S.; Hawes, M.; Perros, M.; Tran, I.; Price, D. A; Quinton, F. J.; Selby, M. D.; Stupple, P. A.; Webster, R.; Wood, A. Bioorg. Med. Chem. Lett. 2009, 19, 5857.

[51] Leeson, P. D.; Springthorpe, B. Nat. Rev. Drug Discovery 2007, 6, 881 . 